\title{
An invariance principle for Azéma martingales
}

\author{
Nathanaël Enriquez \\ Laboratoire de Probabilités de Paris 6, 4, place Jussieu, 75252 Paris cedex 05, France
}

Received 18 January 2006; received in revised form 15 July 2006; accepted 1 October 2006

Available online 31 January 2007

\begin{abstract}
An invariance principle for Azéma martingales is presented as well as a new device to construct solutions of Emery's structure equations.

(C) 2006 Elsevier Masson SAS. All rights reserved.

\section{Résumé}

Nous présentons un principe d'invariance pour les martingales d'Azéma ainsi qu'une nouvelle construction de solutions d'équations de structure d'Emery.
\end{abstract}

(c) 2006 Elsevier Masson SAS. All rights reserved.

MSC: $60 \mathrm{~F} 17 ; 60 \mathrm{G} 44$

Keywords: Invariance principle; Azéma martingales; Structure equations

\section{Introduction}

In [10], Meyer raised the problem of finding "normal" martingales, i.e. martingales $M_{t}$ on a filtered probability space $\left(\Omega, \mathcal{F}, \mathcal{F}_{t}, P\right)$ satisfying $\langle M, M\rangle_{t}=t$, which would enjoy the chaotic representation property. This property, which is stronger than the previsible representation property, requires the direct sum of the chaoses of $M$ to be equal to the whole space $L^{2}(\Omega)$. The only known examples of such martingales were the Brownian motion and the compensated Poisson process until Emery found in [4] a way to construct a whole family of examples, by introducing the beautiful device of structure equations. Among these examples, one of them had been considered earlier by Azéma in his study of random closed sets in [1], and now, the whole class of these self-similar martingales is usually called Azéma martingales. However, their behavior remains a little mysterious, especially, in the neighbourhood of 0 . We refer the reader to the two lecture notes of Yor [15], and of Mansuy and Yor [9], where a chapter is devoted to the subject.

Inspired by some elementary remarks on renewal processes, we present an invariance principle for Azéma martingales, and propose a new device to construct solutions of Emery's structure equations. The principle of these approximations turns out to be quite unusual and differs from the truncation method one can find in dealing with Lévy processes, or from the discretization scheme already used in the subject by Meyer in [11] to construct solutions

E-mail address: enriquez@ccr.jussieu.fr (N. Enriquez). 
of Emery's structure equations. It is mainly based on the introduction of some randomness in the size of the jumps together with keeping the normal martingale property.

\section{The basic example: the first Azéma martingale}

The aim of this section is to introduce, in a natural way, the device we use in the next section to approximate general Azéma martingales.

Let us start with the first Azéma martingale. It was obtained by Azéma in [1], by projecting a Brownian motion $B_{t}$ starting from 0 , on the filtration of $\operatorname{sign}(B)$. This projection yields the following martingale

$$
X_{t}=\sqrt{\frac{\pi}{2}} \operatorname{sign}\left(B_{t}\right) \sqrt{\left(t-g_{t}\right)}
$$

where $g_{t}=\sup \left\{s: s \leqslant t, B_{s}=0\right\}$, which is, up to a constant, a normal martingale. We refer the reader to Chapter IV of Protter's book [13] for a comprehensive discussion about the martingale property of $X_{t}$. A clear justification of the projection property is given in an article of Azéma and Yor [2]: they use an elegant path decomposition of the Brownian motion on $[0, t]$ involving the Brownian meander, seen as the renormalisation of the last incomplete excursion.

Now, it is well known, from P. Lévy, that $2 X_{t}^{2} / \pi t$ follows the arcsine law. On the other hand, there is an old result of Dynkin [3] presented also in Feller's book ([6], Chapter XIV.3), obtaining arcsine laws from asymptotic waiting times of renewal processes having non-integrable inter-arrival times. Now, the question is: can we associate a martingale to any given renewal process, exactly like the Azéma martingale is associated to the zeroes of the Brownian motion?

The following elementary proposition gives a positive answer to this question:

Proposition 1. Let $X_{1}, X_{2}, \ldots$ be an iid sequence of positive random variables admitting a density function. We denote by $S_{n}:=\sum_{i=1}^{n} X_{i}$, and $N_{t}=\sup \left\{n: S_{n} \leqslant t\right\}$. Now, we denote by $\bar{F}(t)=P\left(X_{1}>t\right)$, the tail distribution function of the $X_{i}$ 's.

Let us now introduce a sequence $\varepsilon_{1}, \varepsilon_{2}, \ldots$ of independent symmetric Bernoulli variables i.e. satisfying

$$
P\left(\varepsilon_{i}=1\right)=\frac{1}{2} \quad \text { and } \quad P\left(\varepsilon_{i}=-1\right)=\frac{1}{2} .
$$

The two following assertions hold:

(i) For every law of $X_{1}$, the process $Z_{t}:=\varepsilon_{N_{t}} /\left(\bar{F}\left(t-S_{N_{t}}\right)\right)$ is a martingale with respect to its natural filtration.

(ii) The martingale $Z_{t}$ is normal if and only if $X_{1}$ follows the distribution of

$$
-\ln U+\frac{1}{2 U^{2}}-\frac{1}{2}
$$

where $U$ denotes a uniform variable on $[0,1]$.

Proof. The proof of (i) is based on the fact that, conditionally on the event $\left(t-S_{N_{t}}=x\right.$ ), the probability for $Z_{t}$ to jump between $t$ and $t+h$ is equal to $-\left(\bar{F}^{\prime}(x) / \bar{F}(x)\right) h+\mathrm{o}(h)$, and if a jump occurs, its expectation is equal to $-\varepsilon_{N_{t}} / \bar{F}$. On the other hand, when there is no jump, the increment of the process, between $t$ and $t+h$, is equal to $-\varepsilon_{N_{t}}\left(\bar{F}^{\prime}(x) /(\bar{F}(x))^{2}\right) h+\mathrm{o}(h)$. As a result, the expectations of the increments in both situations are balanced.

Let us now prove (ii). The martingale is normal if and only if the conditional expectation of the square of the increment of $Z_{t}$ between $t$ and $t+h$ is always equal to $h+\mathrm{o}(h)$. Again, conditionally on the event $\left(t-S_{N_{t}}=x\right)$, the probability for $Z_{t}$ to jump between $t$ and $t+h$ is equal to $-\left(\bar{F}^{\prime}(x) / \bar{F}(x)\right) h+\mathrm{o}(h)$, and if a jump occurs, the expectation of its square is equal to

$$
\frac{1}{2}\left(\left(1+\frac{1}{\bar{F}}\right)^{2}+\left(1-\frac{1}{\bar{F}}\right)^{2}\right)
$$

which is $1+1 / \bar{F}^{2}$. Now, the absence of a jump contributes only in a $\mathrm{o}(h)$ in the expectation of the square of the increment of the process. 
Therefore, the martingale is normal if and only if

$$
-\left(\frac{1}{\bar{F}(x)}+\frac{1}{\bar{F}^{3}(x)}\right) \bar{F}^{\prime}(x)=1 .
$$

This yields

$$
-\ln \bar{F}(x)+\frac{1}{2 \bar{F}^{2}(x)}-\frac{1}{2}=x,
$$

so that the distribution function of $X_{1}$ is the inverse of

$$
x \mapsto-\ln (1-x)+\frac{1}{2(1-x)^{2}}-\frac{1}{2} .
$$

This gives the result.

Remark 1. We might have included in Proposition 1, analogous results concerning the sometimes called "second Azéma martingale" i.e. $\sqrt{\frac{\pi}{2}} \sqrt{t-g_{t}}-l_{t}$, where $l_{t}$ denotes the local time of zero at time $t$. Indeed, for every law of $X_{1}$, $\widetilde{Z}_{t}:=1 /\left(\bar{F}\left(t-S_{N_{t}}\right)\right)-N_{t}$ is a martingale which is normal if and only if $X_{1}$ follows the distribution of $1 /\left(2 U^{2}\right)-1 / 2$.

But we shall not insist on that fact since this second Azéma martingale is not Markovian and does not enter the class of Azéma martingales we will consider in the next section.

Let us denote by $Z_{t}^{(-1)}$ the normal martingale $Z_{t}$ of (ii). We note that $Z_{t}^{(-1)}$ is a Markov process, and more precisely,

Proposition 2. The process $Z_{t}^{(-1)}$ is a Markov process with generator:

$$
L^{(-1)} g(x)=\frac{\frac{1}{2}(g(-1)+g(1))-g(x)+x g^{\prime}(x)}{1+x^{2}} .
$$

Proof. From Proposition 1(ii), we deduce that

$$
-\ln \bar{F}(x)+\frac{1}{2 \bar{F}^{2}(x)}-\frac{1}{2}=x
$$

which implies, by differentiation:

$$
-\frac{\bar{F}^{\prime}(x)}{\bar{F}(x)}=\frac{1}{1+\left(1 / \bar{F}^{2}(x)\right)} .
$$

Hence, the rate of jump of the process at time $t$, which is equal to the value of the function $-\bar{F}^{\prime} / \bar{F}$ at $\left(t-S_{N_{t}}\right)$, is precisely $1 /\left(1+\left(Z_{t}^{(-1)}\right)^{2}\right)$, and the speed of the trajectory at a time $t$ between two jumps is equal to $-\varepsilon_{N_{t}}\left(\bar{F}^{\prime}\left(t-S_{N_{t}}\right)\right) /\left(\bar{F}^{2}\left(t-S_{N_{t}}\right)\right)$ which is precisely $Z_{t}^{(-1)} /\left(1+\left(Z_{t}^{(-1)}\right)^{2}\right)$.

Corollary 1. The process $Z_{t}^{(-1, n)}:=Z_{n t}^{(-1)} / \sqrt{n}$ is a Markov process with generator:

$$
L^{(-1, n)} g(x)=\frac{\frac{1}{2}(g(-1 / \sqrt{n})+g(1 / \sqrt{n}))-g(x)+x g^{\prime}(x)}{1 / n+x^{2}} .
$$

Proof. Consider a differentiable function $g$. Introduce the function $h(x)=g(x / \sqrt{n})$.

The image by $L^{(-1, n)}$ of the function $g$ is given by:

$$
L^{(-1, n)} g=n \cdot\left(L^{(-1)} h\right)(\sqrt{n} \cdot x) .
$$

A direct computation gives the announced expression. 
This last corollary already gives an idea of the proximity between $Z_{t}^{n}$ and the first Azéma martingale whose generator is given by

$$
\operatorname{Lg}(x)= \begin{cases}\frac{g(0)-g(x)+x g^{\prime}(x)}{x^{2}} & \text { if } x \neq 0, \\ \frac{1}{2} g^{\prime \prime}(x) & \text { if } x=0\end{cases}
$$

(see, for instance, [15]).

This is the aim of the next section which is to prove, among other things, the convergence in distribution in the sense of the Skorokhod topology of $Z_{n t}^{(-1)} / \sqrt{n}$ towards the first Azéma martingale.

\section{An invariance principle}

Let us begin with the definition of the Azéma martingales.

Following Emery, let us introduce a real parameter $\beta$. The structure equation

$$
\mathrm{d}[X, X]_{t}=\mathrm{d} t+\beta X_{t^{-}} \mathrm{d} X_{t}
$$

has a unique solution.

The existence was proven in a note of Meyer [11], and the uniqueness of the solution was proven by Emery in [4] (for $\beta<0$ ) and [5] (for $\beta>0$ ). The solution of this structure equation is called the Azéma martingale with parameter $\beta$.

When a jump occurs at time $t$, the value of the process changes by some prescribed factor. More precisely, $X_{t}=(1+\beta) X_{t^{-}}$.

- The case $\beta=0$ corresponds to Brownian motion: the continuous normal martingale.

- The case $\beta=-1$ is solved by the martingale $\left(\operatorname{sign} B_{t}\right) \sqrt{2\left(t-g_{t}\right)}$ mentioned in the previous section.

- The case $\beta=-2$ is solved by Parthasarathy's martingale satisfying $\left|X_{t}\right|=\sqrt{t}$, and who changes of sign according to a Poisson point process with intensity $\mathrm{d} t / 4 t$.

- In the case $\beta \leqslant-2$, the solution, at least starting at a non-null point, is rather easy to define, since $\left|X_{t}\right|$ always goes away from 0. In proving the uniqueness of the solution, Emery in [4] shows how to define the process starting from 0 by using a self-similarity argument and a representation of the process by using a time-changed Poisson process.

For $\beta>-2$, things go in a more complicated way since the process $X_{t}$ reaches 0 in finite time, and the above formal generator cannot be of much help after that time.

Let us mention finally that the problem of the chaotic representation property still remains a challenging open question for parameters $\beta$ which do not belong to $[-2,0]$.

Now, let us turn to our main purpose concerning the statement of an invariance principle for these processes. This question is quite natural, since Azéma martingales are self-similar in the sense that, for all $\lambda>0$ the processes $X_{t}$ and $X_{\lambda^{2} t} / \lambda$ are equal in distribution (one can indeed easily check that the process $X_{\lambda^{2} t} / \lambda$ solves the right structure equation, and conclude by uniqueness in law of the solution).

In order to define the process which will be, in our statement, at the origin of such a limit theorem, we generalize the definition of $Z_{t}^{(-1)}$ introduced in the previous section, and define for all real parameters $\beta$ the Markov process $Z_{t}^{(\beta)}$ having generator:

$$
L^{(\beta)}=\frac{\frac{1}{2}(g((1+\beta) x-1)+g((1+\beta) x+1))-g(x)-\beta x g^{\prime}(x)}{1+\beta^{2} x^{2}} .
$$

Let us denote the process $Z_{t}^{(\beta, n)}:=Z_{n t}^{(\beta)} / \sqrt{n}$. It has generator:

$$
L^{(\beta, n)}=\frac{\frac{1}{2}(g((1+\beta) x-1 / \sqrt{n})+g((1+\beta) x+1 / \sqrt{n}))-g(x)-\beta x g^{\prime}(x)}{1 / n+\beta^{2} x^{2}} .
$$

We can now state our main result: 
Theorem 1. The sequence of processes $Z_{t}^{(\beta, n)}:=Z_{n t}^{(\beta)} / \sqrt{n}$ converges to the Azéma martingale with parameter $\beta$, in the sense of the weak convergence for the Skorokhod topology.

Proof. The image by $L^{(\beta, n)}$, of $x$ and $x^{2}$, being respectively 0 and 1 , we deduce that $Z_{t}^{(\beta, n)}$ are normal martingales. Therefore, by Rebolledo's theorem (see [14], II.3.1), this sequence is tight for the weak convergence in the Skorokhod topology. All we have to prove is that all limiting points of the sequence of processes $Z_{t}^{(\beta, n)}$ satisfy the structure equation (1). The uniqueness in law of the solution of (1) allows to conclude.

Let us define at a jump time $t$ of the process $Z_{t}^{(\beta, n)}$, the symmetric Bernoulli variable $\varepsilon_{t}^{(\beta, n)}$ defined by

$$
Z_{t}^{(\beta, n)}-Z_{t^{-}}^{(\beta, n)}=\beta Z_{t}^{(\beta, n)}+\frac{\varepsilon_{t}^{(\beta, n)}}{\sqrt{n}} .
$$

Let us consider $\left[Z^{(\beta, n)}, Z^{(\beta, n)}\right]_{t}$. (We replace, in the sequel, the superscript $(\beta, n)$ by $n$.)

$$
\begin{aligned}
{\left[Z^{n}, Z^{n}\right]_{t} } & =\sum_{s \leqslant t}\left(Z_{s}^{n}-Z_{s^{-}}^{n}\right)^{2}=\sum_{s \leqslant t}\left(\beta Z_{s^{-}}^{n}+\frac{\varepsilon_{s}^{n}}{\sqrt{n}}\right)\left(Z_{s}^{n}-Z_{s^{-}}^{n}\right) \\
& =\int_{0}^{t} \beta Z_{s^{-}}^{n} \mathrm{~d} Z_{s}^{n}-\int_{0}^{t} \beta Z_{s^{-}}^{n} 1_{\Delta Z_{s}^{n} \neq 0} \mathrm{~d} Z_{s}^{n}+\sum_{s \leqslant t} 1_{\Delta Z_{s}^{n} \neq 0} \frac{\varepsilon_{s}^{n}}{\sqrt{n}}\left(\beta Z_{s^{-}}^{n}+\frac{\varepsilon_{s}^{n}}{\sqrt{n}}\right) .
\end{aligned}
$$

Hence,

$$
\left[Z^{n}, Z^{n}\right]_{t}=\int_{0}^{t} \beta Z_{s^{-}}^{n} \mathrm{~d} Z_{s}^{n}+t-\int_{0}^{t} \frac{\mathrm{d} s}{1+n\left(\beta Z_{s^{-}}^{n}\right)^{2}}+\sum_{s \leqslant t} 1_{\Delta Z_{s}^{n} \neq 0} \frac{\varepsilon_{s}^{n}}{\sqrt{n}}\left(\beta Z_{s^{-}}^{n}+\frac{\varepsilon_{s}^{n}}{\sqrt{n}}\right) .
$$

Our task, now, is to show that the two last terms vanish when $n$ goes to infinity, whereas the other terms converge to their analogous quantity for the limiting process, so that every limiting process will solve the structure equation satisfied by an Azéma martingale, and will therefore be identified with the Azéma martingale with parameter $\beta$.

Let us mention that the convergence of the two first terms (i.e. $\left[Z^{n}, Z^{n}\right]_{t}$ and $\int_{0}^{t} \beta Z_{s^{-}}^{n} \mathrm{~d} Z_{s}^{n}$ ) can be deduced from the general results of Jakubowski, Mémin and Pagès in [7] or also of Kurtz and Protter in [8] (who show explicitly in their paper how the proof of Meyer's construction of Azéma martingales can be done using their result), but this is not the case for the last two terms. As a warm-up, we start with a direct treatment of these two first terms.

We proceed, like in Meyer's proof of the existence of solutions of structure equations, and introduce successively:

- a probability space $(\Omega, \mathcal{G}, Q)$, obtained from the Skorokhod representation theorem, on which a subsequence of processes $X_{s}^{\sigma(n)}$, following the law of an extracted sequence of $Z_{s}^{n}$ converges almost surely in $\lambda \otimes Q$. For ease of reading, we keep writing $Z_{s}^{n}$ instead of $X_{s}^{\sigma(n)}$. In other words, we suppose that, for almost every time $t, Z_{t}^{n}$ converges almost surely.

- a sequence of stopping times $T_{N}^{n}=\inf \left\{t:\left|Z_{t}^{n}\right|>N\right\}$ where $N$ is large enough, so that $Q\left(T_{N}^{n} \leqslant t\right)$ is smaller than any prescribed $\epsilon$, uniformly in $n$ (Doob's inequality makes it possible to do so, since the processes $Z_{t}^{n}$ have the same variance).

Let us compare, in a first step, $\left[Z^{n}, Z^{n}\right]_{T_{N}^{n} \wedge t}$ with the sum of the square of its increments along some subdivision $\left(t_{i}\right)$ of the interval $[0, t]$ :

$$
\left[Z^{n}, Z^{n}\right]_{T_{N}^{n} \wedge t}-\sum_{i}\left(Z_{T_{N}^{n} \wedge t_{i+1}}^{n}-Z_{T_{N}^{n} \wedge t_{i}}^{n}\right)^{2}=2 \sum_{i} \int_{t_{i}}^{t_{i}+1}\left(Z_{T_{N}^{n} \wedge s_{-}}^{n}-Z_{T_{N}^{n} \wedge t_{i}}^{n}\right) \mathrm{d} Z_{T_{N}^{n} \wedge s}^{n} .
$$

The compensator of $Z_{T_{N}^{n} \wedge s}$ being equal to $T_{N}^{n} \wedge s$, we deduce,

$$
E\left[\left(\left[Z^{n}, Z^{n}\right]_{T_{N}^{n} \wedge t}-\sum_{i}\left(Z_{T_{N}^{n} \wedge t_{i+1}}^{n}-Z_{T_{N}^{n} \wedge t_{i}}^{n}\right)^{2}\right)^{2}\right] \leqslant 4 E\left[\sum_{i} \int_{t_{i}}^{t_{i+1}}\left(Z_{T_{N}^{n} \wedge s_{-}}^{n}-Z_{T_{N}^{n} \wedge t_{i}}^{n}\right)^{2} \mathrm{~d} s\right]
$$


which in turn is smaller than

$$
4 E\left[\sum_{i} \int_{t_{i}}^{t_{i+1}}\left(Z_{T_{N}^{n} \wedge s_{-}}^{n}-Z_{T_{N}^{n} \wedge t_{i}}^{n}\right)^{2} \mathrm{~d} s\right] \leqslant \frac{4}{3} \sum_{i}\left(t_{i+1}-t_{i}\right)^{3} .
$$

(In this first step, stopping the martingales at $T_{N}^{n}$ just ensures the integrability we need. In the further steps, this stopping argument will be more widely used.)

As a conclusion, we deduce that the "discrete quadratic Riemann sums" of $Z^{n}$ approximate $\left[Z^{n}, Z^{n}\right]$ uniformly, in probability. Now, since the corresponding sums for $Z$ approximate $[Z, Z]$ in probability, it just remains to notice that we can choose the time of our subdivisions among the times $t$ for which $Z_{t}^{n}$ converges almost surely to $Z_{t}$ so that the quadratic sums of $Z^{n}$ will converge almost surely and therefore in probability to their corresponding analog for the process $Z$.

Furthermore, the convergence of the second term $\int_{0}^{t} Z_{s^{-}}^{n} \mathrm{~d} Z_{s}^{n}$ to $\int_{0}^{t} Z_{s^{-}} \mathrm{d} Z_{s}$ is obtained exactly the same way as in Meyer's proof.

Now, we have to prove the convergence to 0 in probability of both remaining terms

$$
\int_{0}^{t} \frac{\mathrm{d} s}{1+n\left(\beta Z_{s^{-}}^{n}\right)^{2}} \text { and } \sum_{s \leqslant t} 1_{\Delta Z_{s}^{n} \neq 0} \frac{\varepsilon_{s}^{n}}{\sqrt{n}}\left(\beta Z_{s^{-}}^{n}+\frac{\varepsilon_{s}^{n}}{\sqrt{n}}\right) .
$$

Again, it suffices to prove it for these quantities when $t$ is replaced by $T_{N}^{n} \wedge t$.

This result will be a consequence of the following key lemma, which gives some uniform control (in $n$ ) on the time spent by the processes $Z_{t}^{n}$ near the origin, and on the number of their jumps:

Lemma 1. The two following convergence results hold: for all $N>0$, and all $t>0$,

(i) $\forall \epsilon>0, \exists \delta>0, \exists n_{0} \in \mathbb{N}$, such that $\forall n \geqslant n_{0}$,

$$
P\left(\int_{0}^{T_{N}^{n} \wedge t} 1_{Z_{s}^{n} \in[-\delta, \delta]} \mathrm{d} s>\epsilon\right)<\epsilon,
$$

(ii) $N_{T_{N}^{n} \wedge t}^{n} / n$ converges to 0 in probability.

Proof. We start with (i). We will use the following notation for a constant which will appear repeatedly: $C=$ $\max (1,1+|\beta|)$.

Let us fix $\varepsilon>0$, and, for some $\delta \in] 0, \frac{N}{2 C}[$, consider the successive stopping times:

- $\tau_{1}^{n}=\inf \left\{t:\left|Z_{t}^{n}\right|>2 \delta\right\}$,

- $\tau_{2}^{n}=\inf \left\{t>\tau_{1}^{n}:\left|Z_{t}^{n}\right|<\delta\right.$ or $\left.\left|Z_{t}^{n}\right|>N\right\}$,

- If $\tau_{2}^{n} \neq T_{N}^{n}$, we define $\tau_{3}^{n}:=\inf \left\{t>\tau_{2}^{n}:\left|Z_{t}^{n}\right|>2 \delta\right\}$

and more generally for $i \geqslant 2$,

- $\tau_{2 i}^{n}:=\inf \left\{t>\tau_{2 i-1}^{n}:\left|Z_{t}^{n}\right|<\delta\right.$ or $\left.\left|Z_{t}^{n}\right|>N\right\}$,

- If $\tau_{2 i}^{n} \neq T_{N}^{n}$, then $\tau_{2 i+1}^{n}:=\inf \left\{t>\tau_{2 i}^{n}:\left|Z_{t}^{n}\right|>2 \delta\right\}$.

Let us denote by $K$, the random integer $K:=\inf \left\{i \geqslant 1: \tau_{2 i}^{n}=T_{N}^{n}\right\}$.

From these definitions, we get obviously:

$$
\int_{0}^{T_{N}^{n} \wedge t} 1_{Z_{s}^{n} \in[-\delta, \delta]} \mathrm{d} s \leqslant \tau_{1}^{n}+\left(\tau_{3}^{n}-\tau_{2}^{n}\right)+\cdots+\left(\tau_{2 K-1}^{n}-\tau_{2 K-2}^{n}\right) .
$$


Now, we can bound the expectation of the right-hand term, by first noticing that, by the optional sampling theorem applied to the martingale $\left(Z_{t}^{n}\right)^{2}-t$,

$$
\begin{aligned}
\forall i \geqslant 1, \quad E\left[\tau_{2 i+1}^{n}-\tau_{2 i}^{n} \mid K>i\right] & =E\left[\left(Z_{\tau_{2 i+1}^{n}}^{n}\right)^{2}-\left(Z_{\tau_{2 i}^{n}}^{n}\right)^{2} \mid K>i\right] \\
& \leqslant E\left[\left(Z_{\tau_{2 i+1}^{n}}^{n}\right)^{2} \mid K>i\right] \leqslant 4 \max \left(1,(1+\beta)^{2}\right) \delta^{2}=4 C^{2} \delta^{2} .
\end{aligned}
$$

And secondly, we can estimate $P(K=i+1 \mid K>i)$, by exploiting that

$$
E\left[Z_{\tau_{2 i+2}^{n}}^{n} \mid K>i\right]=E\left[Z_{\tau_{2 i+1}^{n}}^{n} \mid K>i\right] .
$$

But, on one hand, $\left|Z_{\tau_{2 i+1}^{n}}^{n}\right| \geqslant 2 \delta$ and, on the other hand, $E\left[Z_{\tau_{2 i+2}^{n}}^{n} \mid K>i\right]$ decomposes into $E\left[1_{K=i+1} Z_{\tau_{2 i+2}^{n}}^{n}\right]+$ $E\left[1_{K>i+1} Z_{\tau_{2 i+2}^{n}}^{n}\right]$, with

$$
\left|E\left[1_{K=i+1} Z_{\tau_{2 i+2}^{n}}^{n}\right]\right| \leqslant N C \cdot P(K=i+1 \mid K>i)
$$

and

$$
\left|E\left[1_{K>i+1} Z_{\tau_{2 i+2}^{n}}^{n}\right]\right| \leqslant \delta .
$$

Therefore,

$$
P(K=i+1 \mid K>i) \geqslant \frac{\delta}{N C}
$$

which implies that $P(K \geqslant i) \leqslant\left(1-\frac{\delta}{N C}\right)^{i-1}$.

Now, writing

$$
E\left[\tau_{1}^{n}+\left(\tau_{3}^{n}-\tau_{2}^{n}\right)+\cdots+\left(\tau_{2 K-1}^{n}-\tau_{2 K-2}^{n}\right)\right]=\sum_{i \geqslant 1} E\left[\left(\tau_{2 i-1}^{n}-\tau_{2 i-2}^{n}\right) 1_{K \geqslant i}\right]
$$

and, using the strong Markov property at time $\tau_{2 i-1}^{n}$, we get

$$
E\left[\tau_{1}^{n}+\left(\tau_{3}^{n}-\tau_{2}^{n}\right)+\cdots+\left(\tau_{2 K-1}^{n}-\tau_{2 K-2}^{n}\right)\right] \leqslant 4 C^{2} \delta^{2} \sum_{i \geqslant 1}\left(1-\frac{\delta}{N C}\right)^{i-1}=4 N C^{3} \delta .
$$

We just have to choose $\delta$ such that $4 N C^{3} \delta=\varepsilon^{2}$ and use Markov inequality, to get (i).

To prove (ii), we will strongly exploit (i). To make the reading easier, we will fix $t$ equal to 1 . Let us divide the interval $[0, t]=[0,1]$ into $n$ intervals of the type $I_{k}^{n}=\left[\frac{k}{n}, \frac{k+1}{n}\right]$, where $0 \leqslant k \leqslant n-1$.

Now, $\varepsilon$ being fixed, we introduce $\delta$ like in (i), and define the stopping times:

$$
\sigma_{k}^{n}=\inf \left\{s \in I_{k}^{n},\left|Z_{s}^{n}\right|>\delta\right\}
$$

with $\sigma_{k}^{n}=\infty$ if $\forall s \in I_{k}^{n},\left|Z_{s}^{n}\right| \leqslant \delta$.

From (i), we get that, for $n$ large enough, with probability bigger than $1-\varepsilon$, for more than $(1-\varepsilon) n$ integers $k$, between 0 and $n-1$, we have $\sigma_{k}^{n}<\infty$.

Let us introduce now the stopping time $\tau_{k}^{n}:=\inf \left\{s>\sigma_{k}^{n},\left|Z_{s}^{n}-Z_{s-}^{n}\right| \neq 0\right\}$, with $\tau_{k}^{n}=\infty$ whenever $\sigma_{k}^{n}=\infty$.

Now, for $n$ large enough, the quantity $P\left(\left|\tau_{k}^{n}-\sigma_{k}^{n}\right|<\frac{2}{n} \mid \sigma_{k}^{n}<\infty\right)$ can be bounded uniformly in $k$ by some sequence depending on $n$, which converges to 0 . Indeed, if at some time $s,\left|Z_{s}^{n}\right|>\delta$, and if moreover there is no jump between $s$ and $s+\frac{2}{n}$, then $\left|Z_{s}^{n}\right|$ remains bigger than $\delta / 2$ for $n$ large enough. Now, on the time interval $\left[s, s+\frac{2}{n}\right]$, the intensity of the jump remains smaller than $1 /\left(\beta^{2}(\delta / 2)^{2}\right)$.

We deduce that, conditionally on the event $\left(\sigma_{k}^{n}<\infty\right),\left|\tau_{k}^{n}-\sigma_{k}^{n}\right| \wedge \frac{2}{n}$ is stochastically bigger than $\mathbf{e}_{4 /\left(\beta^{2} \delta^{2}\right)} \wedge \frac{2}{n}$, where $\mathbf{e}_{4 /\left(\beta^{2} \delta^{2}\right)}$ is an exponential variable having parameter $4 /\left(\beta^{2} \delta^{2}\right)$.

So that, for $n$ large enough,

$$
P\left(\left|\tau_{k}^{n}-\sigma_{k}^{n}\right|>\frac{2}{n} \mid \sigma_{k}^{n}<\infty\right)>\exp \left(-\frac{8}{\beta^{2} \delta^{2} n}\right) .
$$

Now, $\left|\tau_{k}^{n}-\sigma_{k}^{n}\right|>\frac{2}{n}$ implies that there is no jump inside the time interval $I_{k+1}^{n}$. 
So, we deduce that, for $n$ large enough, with probability bigger than $1-2 \varepsilon$, the process $Z_{s}^{n}$ will not jump on more than $(1-2 \varepsilon) n$ intervals among the $I_{k}^{n}$ 's.

Finally, since the rate jump of the process is permanently less than $n$ and the length of each $I_{k}^{n}$ is equal to $\frac{1}{n}$, for $n$ large enough, we get that, with an arbitrarily high probability, there are not more than $4 \varepsilon n$ jumps on the remaining $2 \varepsilon n$ intervals $I_{k}^{n}$ which may have some jumps.

End of the proof of Theorem 1. The (i) part of Lemma 1 gives immediately the convergence in probability to 0 , of $\int_{0}^{T_{N}^{n} \wedge t} 1 /\left(1+n\left(\beta Z_{s^{-}}^{n}\right)^{2}\right) \mathrm{d} s$.

Now,

$$
\sum_{s \leqslant T_{N}^{n} \wedge t} 1_{\Delta Z_{s}^{n} \neq 0} \frac{\varepsilon_{s}^{n}}{\sqrt{n}}\left(\beta Z_{s^{-}}^{n}+\frac{\varepsilon_{s}^{n}}{\sqrt{n}}\right)=\frac{N_{T_{N}^{n} \wedge t}^{n}}{n}+\beta \sum_{s \leqslant T_{N}^{n} \wedge t} 1_{\Delta Z_{s}^{n} \neq 0} \frac{\varepsilon_{s}^{n}}{\sqrt{n}} Z_{s^{-}}^{n} .
$$

Part (i) of Lemma 1 implies the convergence to 0 of the first term of this sum, and we bound the $L^{2}$-norm of the second term:

$$
E\left[\left(\sum_{s \leqslant T_{N}^{n} \wedge t} 1_{\Delta Z_{s}^{n} \neq 0} \frac{\varepsilon_{s}^{n}}{\sqrt{n}} Z_{s^{-}}^{n}\right)^{2}\right]=\frac{1}{n} E\left[\sum_{s \leqslant T_{N}^{n} \wedge t} 1_{\Delta Z_{s}^{n} \neq 0}\left(Z_{s^{-}}^{n}\right)^{2}\right] \leqslant N^{2} E\left[\frac{N_{T_{N}^{n} \wedge t}^{n}}{n}\right] .
$$

Now, for all $\varepsilon>0$,

$$
E\left[\frac{N_{T_{N}^{n} \wedge t}^{n}}{n}\right] \leqslant \varepsilon+E\left[\frac{N_{T_{N}^{n} \wedge t}^{n}}{n} 1_{\left(N_{T_{N}^{n} \wedge t}^{n} / n>\varepsilon\right)}\right] \leqslant \varepsilon+E\left[\left(\frac{N_{T_{N}^{n} \wedge t}^{n}}{n}\right)^{2}\right]^{1 / 2} P\left(\frac{N_{T_{N}^{n} \wedge t}^{n}}{n}>\varepsilon\right)^{1 / 2} .
$$

Now, coupling the jump times of the process $Z_{t}^{n}$ with the points of a Poisson process with intensity $n$, we are able to bound $E\left[\left(N_{T_{N}^{n} \wedge t}^{n} / n\right)^{2}\right]$ uniformly in $n$, by $t^{2}$.

We conclude that

$$
\sum_{s \leqslant T_{N}^{n} \wedge t} 1_{\Delta Z_{s}^{n} \neq 0} \frac{\varepsilon_{s}^{n}}{\sqrt{n}}\left(\beta Z_{s^{-}}^{n}+\frac{\varepsilon_{s}^{n}}{\sqrt{n}}\right)
$$

converges to 0 in probability, and obtain Theorem 1 .

Remark 2. We might have replaced the randomness of the jumps, having distribution $\frac{1}{2}\left(\delta_{-1}+\delta_{1}\right)$ in the case of the process $Z_{t}^{(\beta, 1)}$, by any other one, having compact supported distribution with mean 0 and variance 1 . In other words, the result of Theorem 1 remains valid, if we take, instead of $Z_{t}^{(\beta, 1)}$, any process having generator

$$
\frac{\left(\int_{\mathbb{R}} g((1+\beta) x+y) \mu(\mathrm{d} y)\right)-g(x)-\beta x g^{\prime}(x)}{1+\beta^{2} x^{2}}
$$

where $\mu$ is a compact supported measure, with mean 0 and variance 1 . Indeed, one can easily check that we still deal with normal martingales.

\section{An extension to structure equations}

Actually, Meyer is able to construct solutions of the more general structure equation

$$
\mathrm{d}[X, X]_{t}=\mathrm{d} t+f\left(X_{t^{-}}\right) \mathrm{d} X_{t}
$$

where $f$ is an arbitrary continuous function.

The strategy of Meyer consists in solving a discretized structure equation, which leads to a cascade of second degree polynomial equations satisfied by the discretized increments, having always exactly two solutions of opposite signs, which are chosen with respect to the (unique) probability preserving the martingale property of the constructed discrete process. 
We want to show in this section how our approach can be used to exhibit a solution of the structure equation (which may be different from Meyer's in the non-uniqueness cases). Inspired by the processes of previous section, we introduce a sequence of normal martingales $Z_{t}^{(f, n)}$ whose limiting points in the sense of the weak convergence for the Skorokhod topology will satisfy the structure equation (2).

However, although our approach has the advantage to give an invariance principle in the self-similar case of previous section, nonetheless the construction of structure equations for general continuous functions induces delicate discussions on the time spent near the zeroes of the function $f$, and we did not work them out for a general continuous function $f$. So, our aim, in this section, is more to exemplify things, than to arrive to the most general statement, which might be valid for general continuous functions, but we will let this question open here.

We define $Z_{t}^{(f, n)}$, as the Markov process starting at 0 , and having generator:

$$
L^{(f, n)} g(x)=\frac{\frac{1}{2}(g(x+f(x)+1 / \sqrt{n})+g(x+f(x)-1 / \sqrt{n}))-g(x)-f(x) g^{\prime}(x)}{1 / n+f(x)^{2}} .
$$

Proposition 3. Suppose $f$ is a continuous function with isolated zeroes. Suppose, in addition, that, in the neighbourhood of each zero $x_{j}$ of $f, f\left(x_{j}+h\right)=\mathrm{o}(\sqrt{h})$.

Then, the limiting points of the sequence of processes $Z_{t}^{(f, n)}$, in the sense of the weak convergence for the Skorokhod topology, satisfy the structure equation (2).

Proof. Like in the previous section, we start by noticing that the image by $L^{(f, n)}$, of $x$ and $x^{2}$, is respectively 0 and 1 . Indeed,

$$
\frac{1}{2}\left(\left(x+f(x)+\frac{1}{\sqrt{n}}\right)+\left(x+f(x)-\frac{1}{\sqrt{n}}\right)\right)-x-f(x)=0
$$

and

$$
\frac{1}{2}\left(\left(x+f(x)+\frac{1}{\sqrt{n}}\right)^{2}+\left(x+f(x)-\frac{1}{\sqrt{n}}\right)^{2}\right)-x^{2}-2 x f(x)=\frac{1}{n}+f(x)^{2} .
$$

We deduce that $Z_{t}^{(f, n)}$ are normal martingales. Therefore, by Rebolledo's theorem (see [14], II.3.1), this sequence is tight for the weak convergence in the Skorokhod topology.

Now, we follow the proof of Theorem 1, and write:

$$
\left[Z^{n}, Z^{n}\right]_{t}=\int_{0}^{t} f\left(Z_{s^{-}}^{n}\right) \mathrm{d} Z_{s}^{n}+t-\int_{0}^{t} \frac{\mathrm{d} s}{1+n f\left(Z_{s^{-}}^{n}\right)^{2}}+\sum_{s \leqslant t} 1_{\Delta Z_{s}^{n} \neq 0} \frac{\varepsilon_{s}^{n}}{\sqrt{n}}\left(f\left(Z_{s^{-}}^{n}\right)+\frac{\varepsilon_{s}^{n}}{\sqrt{n}}\right) .
$$

Applying again Skorokhod representation theorem, we are able to prove the convergence of the terms $\left[Z^{n}, Z^{n}\right]_{t}$ and $\int_{0}^{t} f\left(Z_{s^{-}}^{n}\right) \mathrm{d} Z_{s}^{n}$ to the analogous quantities for the limiting process.

Now, the key point is to check the analogous property to Part (i) of Lemma 1.

In other words, we have to prove, that, for every $x_{j} \in[-N, N]$ (there are a finite number of them), $\forall \epsilon>0, \exists \delta_{j}>$ $0, \exists n_{0} \in \mathbb{N}$, such that $\forall n \geqslant n_{0}$,

$$
P\left(\int_{0}^{T_{N}^{n} \wedge t} 1_{Z_{s}^{n} \in\left[x_{j}-\delta_{j}, x_{j}+\delta_{j}\right]} \mathrm{d} s>\epsilon\right)<\epsilon .
$$

We then introduce the same stopping times as in Lemma 1, and the differences with the proof of Lemma 1 come out when we estimate $E\left[\tau_{2 i+1}^{n}-\tau_{2 i}^{n} \mid K>i\right]$ :

$$
\begin{aligned}
\forall i \geqslant 1, \quad E\left[\tau_{2 i+1}^{n}-\tau_{2 i}^{n} \mid K>i\right] & =E\left[\left(Z_{\tau_{2 i+1}^{n}}^{n}\right)^{2}-\left(Z_{\tau_{2 i}^{n}}^{n}\right)^{2} \mid K>i\right] \\
& \leqslant E\left[\left(Z_{\tau_{2 i+1}^{n}}^{n}\right)^{2} \mid K>i\right] \leqslant\left(2 \delta_{j}+\sup _{\left[-2 \delta_{j}, 2 \delta_{j}\right]}|f(x)|\right)^{2}=\mathrm{o}\left(\delta_{j}\right)
\end{aligned}
$$

whereas $P(K=i+1 \mid K>i)$ remains bigger than a constant times $\delta_{j}$. 
As a result, $E\left[\tau_{1}^{n}+\left(\tau_{3}^{n}-\tau_{2}^{n}\right)+\cdots+\left(\tau_{2 K-1}^{n}-\tau_{2 K-2}^{n}\right)\right]$ is bounded by a constant times

$$
\frac{\left(2 \delta_{j}+\sup _{\left[-2 \delta_{j}, 2 \delta_{j}\right]}|f(x)|\right)^{2}}{\delta_{j}}=\mathrm{o}(1) .
$$

As in Lemma 1, we choose $\delta_{i}$ such that this fraction is equal to $\varepsilon^{2}$.

We finish the proof the same way as for Theorem 1.

Remark 3. The framework of Proposition 3 includes the case of asymmetric Azéma martingales corresponding to a function $f$ of the form $a x 1_{x \geqslant 0}+b x 1_{x \leqslant 0}$. In this case, Phan proved in [12] that the solution of the structure equation is unique. From this result, we deduce that these processes are self-similar and Proposition 3 provides, in this case, an invariance principle.

Remark 4. Our assumptions in Proposition 3 are obviously not optimal: for instance, if $f$ is null, we are back to the case of a simple random walk, and the convergence to the corresponding solution of the structure equation which is, in this case, the Brownian motion is obviously valid. It would not be hard to check that one can allow $f$ to be null on a locally finite number of intervals, and satisfy the assumptions of Proposition 3 on the complement of the union of these intervals. In this case, the term

$$
\sum_{s \leqslant t} 1_{\Delta Z_{s}^{n} \neq 0} \frac{\varepsilon_{s}^{n}}{\sqrt{n}}\left(f\left(Z_{s^{-}}^{n}\right)+\frac{\varepsilon_{s}^{n}}{\sqrt{n}}\right)
$$

will not vanish in the limit and will "alternatively" contribute with

$$
\int_{0}^{t} \frac{f\left(Z_{s^{-}}^{n}\right)^{2}}{1 / n+f\left(Z_{s^{-}}^{n}\right)^{2}} \mathrm{~d} s
$$

to build a compensator equal to $t$.

It is also very likely that the condition on $f$ to be $\mathrm{o}(\sqrt{h})$ near its zeroes is not optimal (even if our attempts to relax it failed).

\section{Acknowledgements}

It is a pleasure for me to thank here Ashkan Nikeghbali who was at the origin of my interest in the subject, and also Marc Yor and Michel Emery who very kindly initiated me to it.

\section{References}

[1] J. Azéma, Sur les fermés aléatoires (On random closed sets), in: Séminaire de Probabilités, XIX, 1983/84, in: Lecture Notes in Math., vol. 1123, Springer, Berlin, 1985, pp. 397-495.

[2] J. Azéma, M. Yor, Étude d'une martingale remarquable (Study of a remarkable martingale), in: Séminaire de Probabilités, XXIII, in: Lecture Notes in Math., vol. 1372, Springer, Berlin, 1989, pp. 88-130.

[3] E.B. Dynkin, Some limit theorems for sums of independent random variables with infinite mathematical expectations, in: Select. Transl. Math. Statist. and Probability, vol. 1, Inst. Math. Statist. and Amer. Math. Soc., Providence, RI, 1961, pp. 171-189.

[4] M. Emery, On the Azéma martingales, in: Séminaire de Probabilités, XXIII, in: Lecture Notes in Math., vol. 1372, Springer, Berlin, 1989, pp. 66-87.

[5] M. Emery, Sur les martingales d'Azéma (suite) (On Azéma martingales (continued)), in: Séminaire de Probabilités, XXIV, 1988/89, in: Lecture Notes in Math., vol. 1426, Springer, Berlin, 1990, pp. 442-447.

[6] W. Feller, An Introduction to Probability Theory and Its Applications, vol. II, second ed., John Wiley \& Sons, New York, 1971.

[7] A. Jakubowski, J. Mémin, G. Pagès, Convergence en loi des suites d'intégrales stochastiques sur l'espace $D^{1}$ de Skorokhod (Convergence in law of sequences of stochastic integrals on the Skorokhod space D), English summary, Probab. Theory Related Fields 81 (1) (1989) $111-137$.

[8] T. Kurtz, P. Protter, Weak limit theorems for stochastic integrals and stochastic differential equations, Ann. Probab. 19 (3) (1991) 1035-1070.

[9] R. Mansuy, M. Yor, Random times and enlargements of filtrations in a Brownian setting, Lecture Notes in Math., vol. 1873, Springer-Verlag, 2005 . 
[10] P.-A. Meyer, Eléments de probabilités quantiques. I-V (Elements of quantum probabilities. I-V), in: Séminaire de Probabilités, XX, 1984/85, in: Lecture Notes in Math., vol. 1204, Springer, Berlin, 1986, pp. 186-312.

[11] P.-A. Meyer, Construction de solutions d'équations de structure (Construction of solutions of "structure equations"), in: Séminaire de Probabilités, XXIII, in: Lecture Notes in Math., vol. 1372, Springer, Berlin, 1989, pp. 142-145.

[12] A. Phan, Martingales d'Azéma asymétriques. Description élémentaire et unicité (Asymmetric Azéma martingales, elementary description and uniqueness), in: Séminaire de Probabilités, XXXV, in: Lecture Notes in Math., vol. 1755, Springer, Berlin, 2001, pp. 48-86.

[13] P. Protter, Stochastic Integration and Differential Equations, second ed., Stochastic Modelling and Applied Probability, vol. 21, SpringerVerlag, Berlin, 2004.

[14] R. Rebolledo, La méthode des martingales appliquée à l'étude de la convergence en loi de processus, Bull. Soc. Math. France Mém. 62 (1979), $\mathrm{v}+125 \mathrm{pp}$.

[15] M. Yor, Some Aspects of Brownian Motion. Part II. Some Recent Martingale Problems, Lectures in Mathematics ETH Zürich, Birkhäuser, Basel, 1997, xii+144 pp. 